\title{
PET Image Segmentation Based on Gabor Annulus Filtering and Region Growing
}

\author{
P. S. Arya Devi and M. G. Mini
}

\begin{abstract}
Positron emission tomography (PET) image segmentation is a difficult task due to low resolution, high contrast, large variability of pathology, high uncertainties in object boundaries and the inherent noise of PET images. A two staged automatic segmentation technique based on Gabor Annulus filtering and region growing is proposed here. Gabor Annulus filtering is applied on preprocessed image and the result is the input for region growing based on automatic seed selection and threshold determination. Unsupervised evaluation schemes are used for appraising the proposed technique. 93.5\% of the lesions considered for validation have been obtained correctly in the segmented image.
\end{abstract}

Index Terms-Gabor annulus filtering, intra-inter disparity, intra region uniformity, inter region contrast, positron emission tomography, region growing.

\section{INTRODUCTION}

Positron emission tomography (PET) is a nuclear medicine medical imaging technique that can visualize pathologies at a finer molecular level. The image formation is based on the detection of photons emitted from the patient after the injection of radioisotopes like fluorodeoxyglucose (FDG), which have different rates of intake for different tissues [1]. The clear localisation of regions of metabolism in brain and heart, identification and sizing of heart infracts and quantitative permeability measurements in brain tissue are main leverages of PET modality. In addition to early diagnosis, PET imaging finds its application in the evaluation of any drug by identifying and measuring regional metabolic changes before and after drug therapy. The exact location and extent of the lesion are measured by detecting early biochemical changes and checking metabolic activity of targeted tissue. The biochemical process underlying the abnormality of an organ can be accurately assessed with interpretation of good quality PET images [2].

The inherent noise in the PET images, provide less quantitative information about the qualitative features that can be perceived by experienced medical practitioners. The image regions should be delineated to identify and quantify information from PET images. The segmentation of PET images becomes a difficult task due to the inherently poor spatial resolution and signal to noise ratio (SNR). The physical degrading effects such as scatter, attenuation, partial volume effect, and patient motion during scanning add further

Manuscript received January 20, 2015; revised June 16, 2015.

P. S. Arya Devi is with Department of Electronics, Model Engineering College, Kochi, Kerala, India (e-mail: aryaps@mec.ac.in).

M. G. Mini is with College of Engineering, Cherthala, Kerala, India (email: mininair@mec.ac.in). complications to the segmentation task [3]. The intensity range of PET images is large and the intensity values depend heavily upon clinical factors like injection time and dose of radioisotopes and patient factors like tissue uptake and body weight. Normal tissues also uptake different levels of isotope and sometimes these uptake can be more than the tissues with lesions. Thus there can be an overlap in the intensity range of normal and abnormal tissue, which makes the segmentation more difficult [4].

The majority of work performed on PET image segmentation utilises thresholding. Amira et al. suggested a method for PET segmentation utilising the Bayesian information criterion (BIC), Expectation maximisation (EM) and multi-scale Markov model to model spatial correlations between neighbouring image voxels [5]. Hsu et al. proposed Poisson Gradient Vector Flow (PGVF) with genetic algorithm (GA) to automatically find the contour of liver in the PET images [6].

$\mathrm{Li}$ et al. put forward the idea of a two-stage method integrating the adaptive region-growing algorithm and the dual-front active contour model [7]. Spectral clustering and graph based segmentation proposed by Bagci $e t$ al., and Yang and Grigsby mitigate the difficulty of segmenting complex boundaries in low contrast images and are found to be superior to the existing thresholding approaches in PET image segmentation [8], [9]. Abdoli et al. presented a deformable active contour model based on the method proposed by Chan and Vese and obtained more accurate tumour volume delineation from PET images [10].

Zeng et al. proposed volumes of interest (VOI) segmentation method which employs a hierarchical approach combining improved local and global intensity active surface modelling and alpha matting, and achieved sub-voxel segmentation accuracy [11]. Foster et al. propounded a segmentation algorithm based on clustering approach with the affinity propagation (AP) to segment PET images for quantification of tuberculosis in small animal models [12]. A review conducted by Foster et al. on the state-of-the-art image segmentation methods for PET scans of body images, as well as the recent advances in PET image segmentation techniques, pointed resolution related issues, noise and large variability in the shape, texture, and location of pathologies as factors that significantly affect PET image segmentation. They also indicate to the lack of standardization between different segmentation techniques and the need for a publicly available database of PET images for evaluating new and old methods [13].

Zaidi and Naqa [14] conducted a literature survey and categorised various PET image segmentation methodologies as image thresholding methods, variational approaches, learning methods and stochastic modelling-based techniques. 
Manual methods have time consumption as their major impediment, while hard decision making and sensitivity to motion artefacts attributes to the constraints in thresholding techniques. Variational approaches are sensitive to image noise whereas computational complexity and challenges in feature selection offer limitations to learning methods. Even though stochastic modelling techniques can deal with noise in the PET images, the main concerns are the effect of initialization and convergence to local optimal solutions. This suggests for combination of different methodologies to have a good and effective segmentation [14].

In this paper we introduce a two-stage segmentation technique for PET images employing Gabor annulus filtering and region growing. The image to be segmented is enhanced after first pre-processing it for noise removal. The resultant image is filtered using a Gabor annulus kernel. After a series of morphological operations on the filtered image, we get first level of segmentation. This segmented image is subjected to region growing for which seed point and threshold are automatically determined which produces the final segmented image.

\section{THEORY}

\section{A. Gabor Annulus}

Gabor wavelets have the calibre to identify image features and patterns at definite scales and orientations. This expertise is employed in Gabor Annulus approach to detect circular features. In this technique, the traditional Gabor filter is offset by a radius and encloses the origin. It is defined as

$$
G(x, y)=\frac{1}{2 \pi \sigma r_{0}} e^{-\pi\left[\frac{\left(r-r_{0}\right)^{2}}{\sigma^{2}}\right]} e^{i\left[2 \pi f_{0}\left(r-r_{0}\right)\right]}
$$

where $r=\sqrt{\left(x-x_{0}\right)^{2}+\left(y-y_{0}\right)^{2}}$

Here $\sigma$ represents the standard deviation of the Gaussian envelope in the waveform direction. The envelope expands radially outwards from the centre of the filter which is specified by coordinates $\left(x_{0}, y_{0}\right)$ and $r_{0}$ denotes the radius of the 'annulus' shape.

The filter responds to circular image features made up of matching radial frequencies on their boundaries. The centre of the circular image feature is identified by finding the location where the filter response will be the strongest. The Gabor Annulus technique creates a family of filters at various scales which can be utilised to match circular image features at various sizes within the image. The filters are radial symmetric so that rotations need not be created at different orientations [15].

\section{B. Region Growing}

Region growing can be considered both as region-based image segmentation method and pixel-based image segmentation method. The neighbouring pixels of initial 'seed points' are analysed to decide whether the pixel neighbours should be added to the region. Region-growing approaches exploit the important fact that pixels which are close together have similar gray values [16]. In region growing, an initial set of small areas is iteratively merged according to similarity constraints. The steps involved in region growing are

1) Start by choosing an arbitrary seed pixel and compare it with neighboring pixels

2) Region is grown from the seed pixel by adding in neighboring pixels that are similar, increasing the size of the region

3) When the growth of one region stops we simply choose another seed pixel which does not yet belong to any region and start again.

4) This whole process is continued until all pixels belong to some region [17].

The selection of seed points can affect segmentation results and different choices of seed points may produce different results [18].

\section{PROPOSED METHOD}

The proposed method is a two staged segmentation technique having Gabor annulus filtering as first stage and region growing with automatic seed selection as the second stage. The original image first undergoes pre-processing in the form of morphological operations and image enhancement. The image enhancement is done by a modified type of high boost filtering. The high pass filtered image which is obtained from the application of stationary wavelet transform (SWT) and modulus maxima on the image is added to the original image for enhancing it [19]. The enhanced image is subjected to Gabor annulus filtering.

The lesions and most of the prominent organs like brain, heart, etc. are almost circular in shape when considering axial slices. So this method is suitable here. The kernel size, standard deviation of Gaussian envelope, wavelength and radius of annulus are determined experimentally. The filtered image is subjected to dilation and edge detection using Laplacian of Gaussian operator (LoG) and operated morphologically upon and boundary is drawn to create the mask for segmentation. This gives the output in the first stage of segmentation.

Another level of segmentation is needed to reduce under-segmentation and we propose region-growing with automatic seed selection. The region is iteratively grown by comparing all unallocated neighbouring pixels to the region. The difference between pixel intensity value and region mean is the measure of similarity chosen. The pixel with smallest difference measured is allocated to the respective region. The process stops when the difference is greater than a particular threshold.

For automatic seed selection, the image is divided into blocks of size $5 \times 5$ and standard deviation of each block is found. The block with largest standard deviation is identified and pixel with maximum value is selected as initial seed point. Threshold determination is another important aspect that has to be taken care of in region growing. Here we use the technique of finding largest pixel values along the column of the image. The average of the values thus obtained is found and rounded off. Half of this value serves as threshold in this case. The mask for second level of segmentation is obtained as a result of performing morphological operations on the result of region growing. 


\section{APPRAISAL NORMS}

The appraisal of segmentation can be subjective or objective. In subjective evaluation the quality of segmentation is decided by a viewer who uses his knowledge and experience for discretion. In objective measures the quality is assessed in numeric values. The objective evaluation can be classified as supervised evaluation and unsupervised evaluation. The supervised evaluation methods evaluate segmentation algorithms by comparing the resulting segmented image against a manually-segmented reference image, which is referred to as a gold standard or ground-truth. The degree of similarity between the human and machine segmented images determines the quality of the segmented image. Unsupervised evaluation methods evaluate a segmented image based on how well it matches a broad set of characteristics of segmented images as desired by humans [20]-[25]. For evaluating proposed segmentation technique, we choose inter-region contrast, intra-region uniformity and combination of intra-region and inter-region disparity suggested by Levine and Nazif [23].

Inter-region contrast is calculated from sum of contrasts of regions $R_{i}$ balanced by their surface areas. The contrast of a region is calculated starting from contrasts with the regions which are conterminous to it. Let $c_{i j}$ be the contrast between two regions $R_{i}$ and $R_{j}$ having $m_{i}$ and $m_{j}$ as their mean gray levels respectively.

$$
c_{i j}=\frac{\left|m_{i}-m_{j}\right|}{m_{i}+m_{j}}
$$

The contrast of the region $R_{i}$ is given as

$$
c_{i}=\sum_{W_{i}} p_{i j} c_{i j}
$$

where $W_{i}$ denotes the indices of the regions adjacent to the region $i$ and $p_{i j}=\frac{l_{i j}}{l_{i}}$, the ratio of the length of the common boundary between $R_{i}$ and $R_{j}$ to the perimeter of $R_{i}$. The interclass contrast is given as

$$
C_{\text {Inter }}=\frac{\sum_{R_{i}} w_{i} c_{i}}{\sum_{R_{i}} w_{i}}
$$

$w_{i}$ is the weight associated to each region, and here it is taken as the area of the region [24].

The uniformity of a feature over a region is inversely proportional to the variance of the values of that feature evaluated at every pixel belonging to that region. A nil value for the variance requires all the pixels to have the exact same value for the feature, while a large variance would indicate a large spread from the mean of the feature value across the region. The intra class uniformity criterion suggested by Levine and Nazif [25] is defined by computing the sum of the normalized standard deviation of each region. Let $C(p)$ be the gray value of pixel $p$. The average gray level value of a region $R$ is $\bar{C}(R)$. Let $S_{i}$ be a region, the average gray level value of the region is given as

$$
\bar{C}\left(S_{i}\right)=\frac{1}{A_{i}} \sum_{p=S_{i}} C(p)
$$

where $A_{i}$ is the number of pixels in the region $S_{i}$. The normalised gray level variance on the region $S_{i}$ is given as:

$$
\sigma^{2}\left(S_{i}\right)=4 * \frac{\frac{1}{A_{i}} \sum_{p=S_{i}}\left(C^{2}(p)-\bar{C}^{2}\left(S_{i}\right)\right)}{\max _{p \in S_{i}}[(p)]-\underset{p \in S_{i}}{\min [C(p)]}}
$$

If the segmented image $I_{s}$ is formed with $N_{s}$ regions, the intra class uniformity is defined as

$$
U_{\text {Intra }}=1-\sum_{i=1}^{N_{S}} \sigma^{2}\left(S_{i}\right)
$$

Intra-inter region disparity is another measure considered for evaluation. It combines with the inter-region dissimilarity and inter-region disparity. This is represented as follows:

$$
D_{\text {Intru-lnter }}=\frac{1+1 / C_{N_{S}^{2}}^{2} \sum_{i, j=1 ; i \neq j}^{N_{S}}\left(\left|\bar{C}\left(S_{i}\right)-\bar{C}\left(S_{j}\right)\right| / 512-4 / 255^{2} N_{S}\right) \sum_{i=1}^{N_{S}} \sigma^{2}\left(S_{i}\right)}{2}
$$

where $C_{N_{S}}^{2}$ is number of combinations of two regions among $N_{S}$. Intra-region disparity is computed by the normalized standard deviation of gray levels in each region. The inter-regions disparity computes the dissimilarity of the average gray level of two regions in the segmentation result [26].

\section{RESULTS AND DISCUSSION}

The database used for this study is Oncopet_DB, a freely available realistic simulated database of whole body 18F-FDG PET images for oncology. The database comprises of 100 images, including 50 normal cases coming from different realizations of noise of the healthy model and 50 pathological cases including lesions of calibrated uptakes and various diameters. A model of lesion extent based on the clinical description of lymphoma patients is used. Lymphoma affects the lymphatic system through the lymph nodes and other organs included in the immune system. It mostly affects young adults and is especially reactive to standard treatments, such as chemotherapy or radiotherapy. PET is used for the crucial part of staging and treatment follow-up of lymphoma, due to a higher sensitivity and specificity than anatomical medical imaging modalities. The small lesions mainly localized in lymph nodes indicate lymphoma and lesions can also extend to other organs such as the liver, the spleen, and the lungs [27].

Each image in this database is of size $128 \times 128 \times 375$. Standardised uptake values (SUV) are widely used to measure FDG uptake. The more reliable SUV normalisation of FDG uptake for the body surface area is used here [28]. To reduce the complexity of computation each of the axial slices is taken one by one and processed. The image after initial pre-processing is subjected to Gabor annulus filtering. The kernel size is determined as 32 while gaussian deviation is found as 4 , the frequency is taken as 400 and radius of annulus 
as 1 is used. After filtering, the image is dilated and morphologically operated upon. The segmentation is appraised using three unsupervised evaluation criteria viz., inter-region contrast (inter), intra-region uniformity (intra) and combination of intra-region and inter-region disparity (intra- inter). This segmented output is fed as input to region growing stage. This stage of segmentation gives better segmentation than single level segmentation. After this level, the three criteria are also calculated.

Fig. 1 shows the result of segmentation of an image having
2 lesions. The two lesions present in the image are indicated by the white arrows. Fig. 1(b) displays segmentation result after Gabor annulus filtering and subsequent segmentation. Fig. 1(c) shows the segmentation result after region growing on the result of first stage which provides a better segmentation in comparison with the first. Fig. 2 shows the result of segmentation on another image having a small lesion. Only partial success is achieved in this case. Fig. 3 shows the result of segmentation on an image having no lesions.

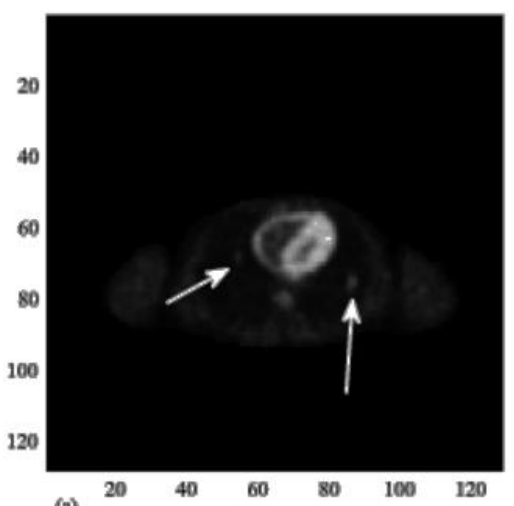
(a)

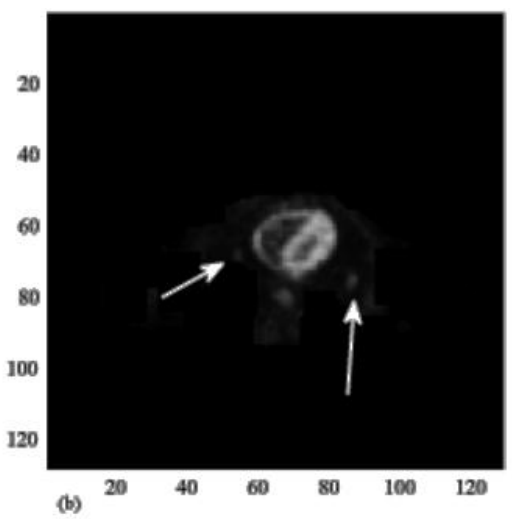

(b) $\begin{array}{llllll}20 & 40 & 60 & 80 & 100 & 120\end{array}$

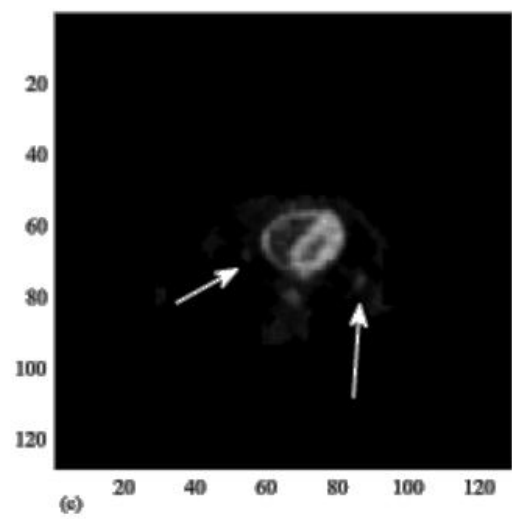

(c) $\begin{array}{rrrrrr}20 & 40 & 60 & 80 & 100 & 120\end{array}$

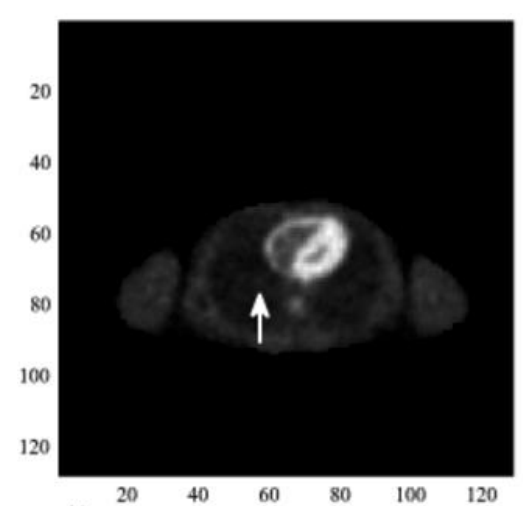

(a)

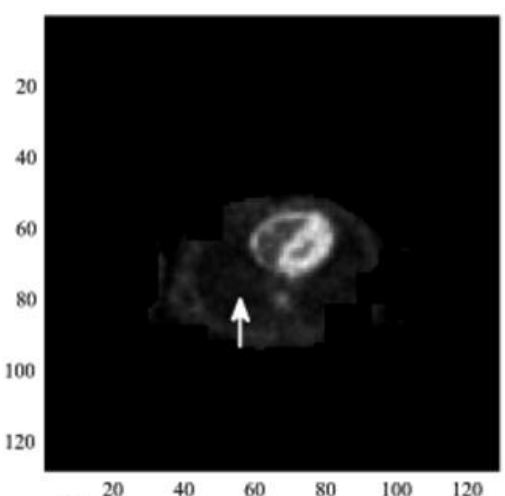

(b)

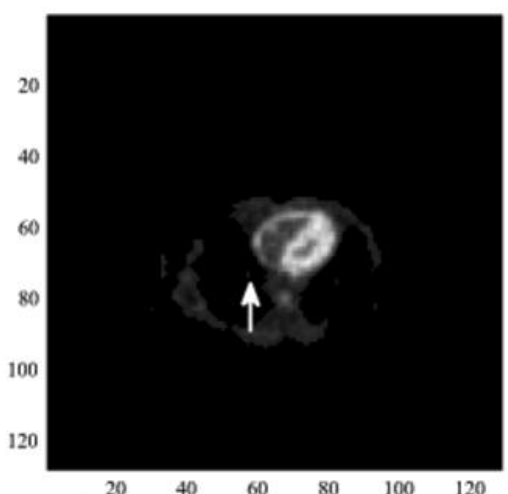

(c)

Fig. 2. . Segmentation of an image with single lesion (a) Original pre-processed image (b) Segmented image after stage 1 and (c) Segmented image after stage 2.
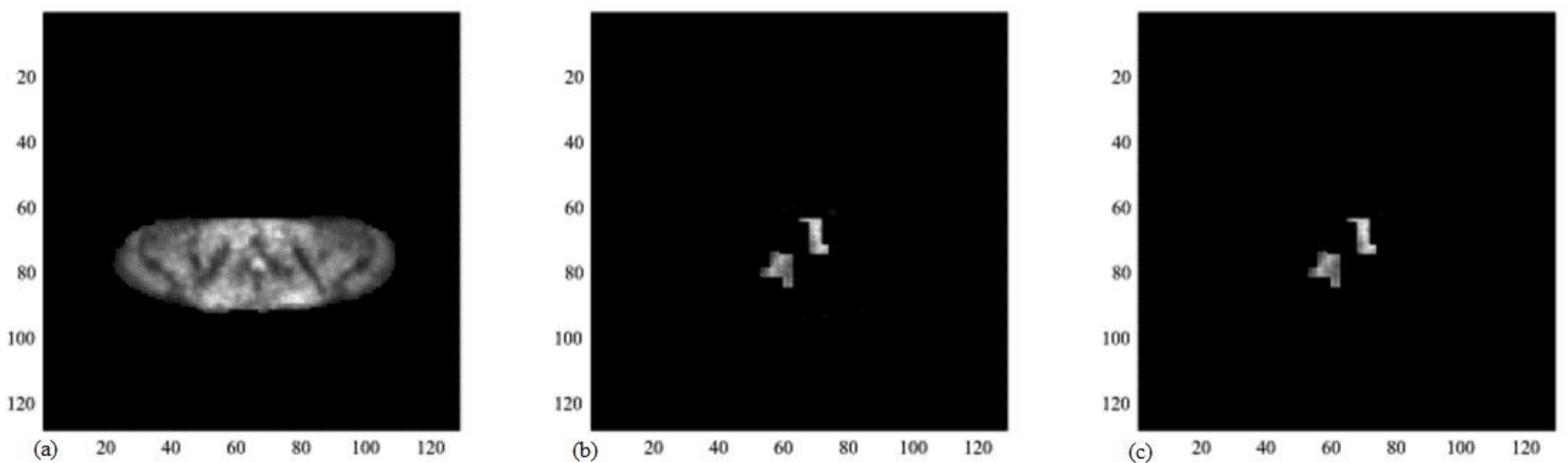

Fig. 3. Segmentation of an image without lesion (a) Original Pre-processed image (b) Segmented image after stage 1 and (c) Segmented image after stage 2 .

The subjective evaluation is performed on 250 axial slices from 39 images for both level of segmentation. The average values for inter, intra and intra-inter are calculated and tabulated in Table I. From the table it is observed that inter value after second level of segmentation is greater which clearly means a better segmentation after the region growing phase. Intra value is slightly better after first level of segmentation while intra-inter value shows improved performance after second level of segmentation. For a better segmentation inter value should be closer to 1 while intra value should be less. Higher intra-inter value indicates that segmentation quality is better.

Out of the 250 slices from different images considered for evaluation, the lesions were segmented correctly in 234 cases. 
In 14 slices, the lesions were missed while in 2 we could partly get lesions in the segmented regions. The partially detected cases have lesions in the first stage and partially lost them in the second stage of segmentation. After the first stage the lesions were missed in 10 slices. In the database examined here the lesions are spread out in blood pool, lungs, liver and spleen. The database consists of 2 categories of images images with 5 lesions (MM_5) and images with 10 lesions (MM_10).

\begin{tabular}{lll}
\multicolumn{2}{l}{ TABLE I: PERFORMANCE MEASURES FOR 2 } & STAGES OF SEGMENTATION \\
\hline \hline Appraisal Norms & SegmentationStage1 & Segmentation Stage 2 \\
\hline Inter $C_{\text {Inter }}$ & 0.8477 & 0.8951 \\
Intra $U_{\text {Intra }}$ & 0.0419 & 0.0444 \\
Intra-Inter $D_{\text {Intra-Inter }}$ & 0.5102 & 0.5230 \\
\hline \hline
\end{tabular}

TABLE II: COMPARISON OF NO. OF LESIONS PRESENT IN ORIGINAL AND SEGMENTED IMAGES

\begin{tabular}{lcccccc}
\hline \hline $\begin{array}{l}\text { Lesion } \\
\text { Location }\end{array}$ & \multicolumn{3}{c}{ No. of Lesions Considered } & \multicolumn{2}{c}{ No. of Lesions Segmented } \\
\hline \hline & MM_5 & MM_10 & Total & MM_5 & MM_10 & Total \\
\hline Blood pool & 73 & 49 & 122 & 71 & 48 & 119 \\
Lungs & 10 & 43 & 53 & 8 & 32 & 40 \\
Liver & 3 & 50 & 53 & 3 & 50 & 53 \\
Spleen & 1 & 21 & 22 & 1 & 21 & 22 \\
\hline \hline
\end{tabular}

The over-all detection rate is $97.54 \%$ for blood pool lesions, $75.47 \%$ for lungs lesions and $100 \%$ for both liver and spleen lesions. The sensitivity of the proposed method is less for lungs lesions while compared to lesions in other locations. This may be due to the fact that the brightness of the lung lesions is comparable to other structures in lungs.

The area of the segmented image in pixels is calculated after first and second stages of the proposed method. An average decrease of $40 \%$ in area is recorded in the comparison of segmented results after two stages. A maximum decrease of $73.46 \%$ is obtained while the minimum decrease in area obtained is $10.54 \%$. Thus with this method under segmentation problem that existed with the single stage segmentation could be solved.

\section{CONCLUSION}

The two-stage segmentation proposed for PET images here exploits the concepts of edge based and region based segmentation effectively. Gabor Annulus filtering and region growing techniques form the backbone of the proposed method. The system is evaluated using three validation criteria viz. inter-region contrast, intra-region uniformity and combination of intra-region and inter-region disparity. The successful lesion capture rate achieved using this technique is $93.8 \%$. The rate of partial success obtained is $0.8 \%$ while the failure rate acquired is $5.6 \%$. The proposed method can be helpful in finding region of interest automatically in PET images.

\section{REFERENCES}

[1] G. Dougherty, Digital Image Processing for Medical Applications, New York, Cambridge Universiy Press, 2009.
[2] P. B. Davis and M. A. Abidi, "Enhancement of PET images," in Proc. SPIE, 1989, vol. 1092, pp. 584-591.

[3] A. Boudraa and P. D. H. Zaidi, "Image segmentation techniques in nuclear medicine imaging," Quantitative Analysis in Nuclear Medicine Imaging, Springer, 2006, pp. 308-357.

[4] H. Guan, T. Kubota, X. Huang, X. S. Zhou, and M. Turk, "Automatic hot spot detection and segmentation in whole body FDG-PET images," in Proc. IEEE International Conference in Image Processing, 2006, pp. $85-88$

[5] A. Amira, S. Chandrasekaran, D. W. Montgomery, and I. S. Uzun, "A segmentation concept for Positron Emission Tomography imaging using multiresolution analysis," Neurocomputing, vol. 71, no. 10, pp. 1954-1965, March 2008.

[6] C.-Y. Hsu, C.-Y. Liu, and C.-M. Chen, "Automatic segmentation of liver PET images," Computerized Medical Imaging and Graphics, vol. 32, no. 7, pp. 601-610, July 2008.

[7] H. Li, W. L. Thorstad, K. J. Biehl, R. Laforest, Y. Su, K. I. Shoghi, E. D. Donnelly, D. A. Low, and W. Lu, “A novel PET tumor delineation method based on adaptive region-growing and dual-front active contours," Medical Physics, vol. 35, no. 8, pp. 3711-3721, Aug. 2008.

[8] U. Bagci, J. Yao, J. Caban, E. Turkbey, O. Aras, and D. J. Mollura, “A graph-theoretic approach for segmentation of PET images," in Proc. Annual International Conference of the IEEE in Engineering in Medicine and Biology Society, EMBC, 2011, pp. 8479-8482.

[9] F. Yang and P. W. Grigsby, "Delineation of FDG-PET tumors from heterogeneous background using spectral clustering," European Journal of Radiology, vol. 81, no. 11, pp. 3535-3541, Nov. 2012.

[10] M. Abdoli, R. Dierckx, and H. Zaidi, "Contourlet-based active contour model for PET image segmentation," Medical Physics, vol. 40, no. 8, pp. 082507-1-082507-12, July 2013.

[11] Z. Zeng, J. Wang, B. Tiddeman, and R. Zwiggelaar, "Unsupervised tumour segmentation in PET using local and global intensity-fitting active surface and alpha matting," Computers in Biology and Medicine, vol. 43, no. 10, pp. 1530-1544, July 2013.

[12] B. Foster, U. Bagci, Z. Xu, B. Dey, B. Luna, W. Bishai, S. Jain, and D. J. Mollura, "Segmentation of PET images for computer-aided functional quantification of tuberculosis in small animal models," IEEE Trans. on BioMedical Engineering, vol. 61, no. 3, pp. 711-724, March 2014

[13] B. Foster, U. Bagci, A. Mansoor, Z. Xu, and D. J. Mollura, "A review on segmentation of Positron Emission Tomography images," Computers in Biology and Medicine, vol. 50, pp. 76-96, April 2014.

[14] H. Zaidi and I. Naqa, "PET-guided delineation of radiation therapy treatment volumes: a survey of image segmentation techniques," European Journal of Nuclear Medicine and Molecular Imaging, vol. 37, no. 11, pp. 2165-2187, March 2010.

[15] A. Rhodes and L. Bai, "Circle detection using a gabor annulus," in Proc. British Conference on Machine Vision, 2011, pp. 108.1-108.11.

[16] A. Ghule and P. Deshmukh, "Image segmentation available techniques, open issues and region growing algorithm," Journal of Signal Processing, vol. 3, March 2012.

[17] R. C. Gonzalez and R. E. Woods, Digital Image Processing, 2nd ed. Prentice Hall India, 2002.

[18] Y. Yang, H. Peng, Y. Jiang, X. Huang, and J. Zhang, "A region-based image segmentation method under P-systems," Journal of Information \& Computational Science, vol. 10, no. 10, July 2013.

[19] P. S. A. Devi and M. G. Mini, "Mammographic Image enhancement based on SWT and high boost filtering," International Journal of Computer Theory and Engineering, vol. 7, no. 5, pp. 374-378, 2015.

[20] H. Zhang, J. E. Fritts, and S. A. Goldman, "Image segmentation evaluation: A survey of unsupervised methods," Computer Vision and Image Understanding, vol. 110, no. 2, pp. 260-280, July 2008.

[21] S. Chabrier, B. Emile, H. Laurent, C. Rosenberger, and P. Marche, "Unsupervised evaluation of image segmentation application to multi-spectral images," in Proc. the 17th International Conference on Pattern Recognition, vol. 1, 2004, pp. 576-579.

[22] S. Chabrier, B. Emile, H. Laurent, C. Rosenberger, and P. Marche, "Evaluating the segmentation result of a gray-level image," in Proc. the 12th European Conference on Signal Processing, 2004, pp. 953-956.

[23] M. D. Levine and A. M. Nazif, "Dynamic measurement of computer generated image segmentations," IEEE Trans. Pattern Analysis and Machine Intelligence, no. 2, pp. 155-164, 1985.

[24] S. P. Foliguet and L. Guigues, "Multi-scale criteria for the evaluation of image segmentation algorithms," Journal of Multimedia, vol. 3, no. 5, pp. 42-56, Dec. 2008.

[25] S. Ouattara, G. L. Loum, A. Cl'ement, and B. Vigouroux, "Analysis of the relevance of evaluation criteria for multicomponent image 
segmentation," Journal of Software Engineering and Applications, vol. 4, no. 6, p. 371, June 2011.

[26] S. Chabrier, B. Emile, C. Rosenberger, and H. Laurent, "Unsupervised performance evaluation of image segmentation," EURASIP Journal on Applied Signal Processing, vol. 2006, pp. 217-217, Jan. 2006.

[27] S. Tome"1, A. Reilhac, D. Visvikis, N. Boussion, C. Odet, F. Giammarile, and C. Lartizien, "Oncopet DB: A freely distributed database of realistic simulated whole body 18F-FDG PET images for oncology," IEEE Trans. Nuclear Science, vol. 57, no. 1, pp. 246-255, Feb. 2010

[28] C. K. Kim, N. C. Gupta, B. Chandramouli, and A. Alavi, "Standardized uptake values of FDG: Body surface area correction is preferable to body weight correction," The Journal of Nuclear Medicine, vol. 35, no. 1, pp. 164-167, Jan. 1994.

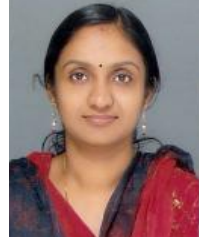

P. S. Arya Devi obtained her B.E. degree in electronics \& communication engineering from Bharatidasan University, received M.Tech. degree in applied electronics from Mahatma Gandhi University, Kerala in 2008. Her research interests include image compression, information theory and coding.

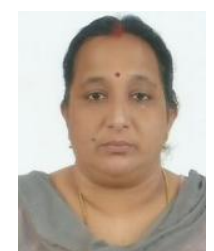

M. G. Mini has received her $\mathrm{PhD}$ degree from CUSAT, Kerala in 2005. Her research interests include digital image processing, VLSI, etc. She has several research papers published in national and international journals. 